\title{
Association between masticatory muscle activity and oral conditions in young female college students
}

\author{
Cha-Young Pyo ${ }^{1}$, Tae-Hoon Kim², Da-Hye $\mathrm{Kim}^{3}$ \\ ${ }^{1}$ Gimhae Jeil Dental Clinic, Gimhae, ${ }^{2}$ Department of Occupational Therapy, Division of Health Sciences, Dongseo University, Busan, ${ }^{3}$ Department of \\ Dental Hygiene, Division of Health Sciences, Dongseo University, Busan, Korea
}

\begin{abstract}
The purpose of this study was to determine the characteristics of masticatory muscle activity and various oral condition factors, and to analyze their associations in order to improve the prevention and diagnosis of masticatory musclerelated diseases. This study included 30 Korean females (mean age of 20 years, age range of 19-21 years). Participants were instructed to complete a self-written questionnaire on factors that may affect their muscle activity. Surface electromyography was used to measure the activity of the masseter and temporalis muscles. We also observed the buccal mucosa ridge, tongue indentation, tooth cracks and fractures, and mandibular tori in the oral cavity. Spearman correlation analysis and the nonparametric Mann-Whitney $U$ test were applied to the data. When subjects had temporomandibular disorder, the right temporalis muscle exhibited significantly lower activity $(P<0.05)$. Those who had received orthodontic treatment within the previous 2 years showed significantly lower activity of the left masseter muscle $(P<0.05)$. Those who had a left buccal mucosa ridge exhibited lower activity of the left masseter muscle and higher activity of the right temporalis muscle compared with those without such a ridge $(P<0.05)$. Participants with no tongue indentation showed significantly higher activity in the left masseter muscle $(P<0.05)$. These results indicate that there are relationships between masticatory muscle activity and various factors related to the oral condition.
\end{abstract}

Key words: Masticatory muscles, Buccal mucosa ridge, Tongue indentation, Tooth cracks, Mandibular torus

Received May 25, 2021; Revised July 12, 2021; Accepted July 26, 2021

\section{Introduction}

The various anatomical structures of the head are interrelated. Among these, the masticatory muscles, mandible, temporomandibular joint (TMJ), teeth, and soft tissues in the mouth have coordinated structures and functions. Certain factors interfering with this coordination can re-

\section{Corresponding author:}

Da-Hye Kim (iD

Department of Dental Hygiene, Division of Health Sciences, Dongseo University, Busan 47011, Korea

E-mail: dahye1124@dongseo.ac.kr sult in certain changes [1]. Masticatory muscles move the mandible to finely chew foods through the teeth occlusions. This muscle group includes the masseter muscle, temporalis muscle, and medial and lateral pterygoid muscles, which are important structures that affect swallowing and digestion [2]. The square masseter muscle is the thickest masticatory muscle, originates in the zygomatic arch, and inserts into the masseteric tuberosity of the mandible. The fan-shaped temporalis muscle originates in the inferior temporal line, temporal fossa, and internal aspect of the deep temporal fascia, and inserts into the coronoid process and anterior border of the mandibular ramus [3]. Each masticatory muscle elevates, depresses, protrudes, retrudes, or laterally translates the mandible according to its position and function.

\section{Copyright (c) 2021. Anatomy \& Cell Biology}

This is an Open Access article distributed under the terms of the Creative Commons Attribution Non-Commercial License (http://creativecommons.org/licenses/by-nc/4.0/) which permits unrestricted non-commercial use, distribution, and reproduction in any medium, provided the original work is properly cited. 
Electrical activity occurs in the muscle fiber during masticatory muscle contraction, and the associated electrical signal generated canbe recorded on electrodes using electromyography. Electromyography is commonly used to measure muscle activities of the upper and lower limbs [4]. In addition, electromyograms are widely used to observe masticatory muscle activity, with bite forces increasing in proportion with the activity of the masticatory muscles [5]. The masseter muscle, which is the most active masticatory muscle, becomes thicker as occlusal forces increase [6]. Excessive development of the masseter muscle creates a square jaw [7]. Takeuchi-Sato et al. [8] suggested that head and face shape are related to the activity of the masticatory muscles. Custodio et al. [9] suggested that the vertical type of the face can affect the maximum occlusal force and activity of the masticatory muscles. Previous studies have analyzed the relationships between activity of the masticatory muscles and other variables based on the above phenomena. However, the associations between activity of the masticatory muscles and oral conditions such as the buccal mucosa ridge, tongue indentation, tooth cracks and fractures, and mandibular torus have not been comprehensively analyzed.

Functional changes in the masticatory muscles can cause various problems. The buccal mucosa ridge is a structure along the position where the upper and lower teeth occlude on the buccal mucosa where they contact the posterior teeth. This is considered to occur due to mechanical stimulation that causes the compression and thickening of the mucosal epithelium [10]. It is a structure associated with bruxism and clenching, and it has been reported as relating to the number and crowding of teeth [11]. Tongue indentation, also known as tongue scalloping, is a structure at the edge of the tongue. Bruxism and clenching have also been reported to influence its development [12]. Mandibular torus occur in proportion to the number of teeth [13], and have been reported to be more prevalent in dentulous than edentulous patients [14]. Mechanical stress (occlusal force) appears to be the main factor in bad oral habits and temporomandibular disorder (TMD), which are considered factors that contribute to the occurrence of mandibular tori $[15,16]$. Bone remodeling is promoted and strengthened when external force is applied [17], and the mandibular torus area is continuously exposed to occlusal stimuli and has sensitive reactions [18]. The presence of a mandibular torus makes forming a suitable inner surface for dentures difficult, and obtaining sufficient marginal closure from the dentures is unlikely.
In addition, excessive contraction of the masticatory muscles can cause problems such as cracked tooth syndrome or TMD, which occurs due to abnormalities in the masticatory muscles $[19,20]$. Cracked tooth syndrome involves the formation of thin cracks on the teeth due to the bite force between the two sets of teeth. It especially occurs when large fillings are present on the teeth, and can be aggravated by bruxism [21]. If it is left untreated, tooth fracture or inflammation can occur, possibly leading to the need for tooth crowning or dental implants after extraction. TMD is a complex disease involving the TMJ, masticatory muscles, musculoskeletal system of the head and neck, and occlusion. It is one of the four main diseases of dentistry, along with dental caries, periodontal disease, and malocclusion [22, 23]. Symptoms that may appear include pain in the TMJ and related muscles, articular sounds during mandible movements, and restricted range of motion [24]. Pain and functional problems in TMD patients are associated not only with abnormalities in the joints themselves, but also with excessive activity of the masticatory muscles. Systematic research on the masticatory muscles is needed to improve the quality of life for affected individuals and to reduce the medical costs of these diseases. The purpose of this study was therefore to determine the characteristics of masticatory muscle activity and various factors related to the oral condition, and to analyze their association and provide basic data with the aim of improving the prevention and diagnosis of masticatory muscle-related diseases.

\section{Materials and Methods}

This study included 30 Korean females (mean age of 20 years, age range of 19-21 years). Approval for this study was obtained from the Institutional Review Board of Dongseo University (No. 1041493-A-2020-011). We explained the anatomical structure of the masticatory muscles, basic principles of surface electromyograms, and our experimental procedures to those who agreed to participate. After this briefing session, the final subjects selected were those who volunteered to participate in the study. Before measuring the surface electromyogram, participants were instructed to complete a self-written questionnaire on factors (e.g., TMD, oral parafunctions, and food habits) that may affect their muscle activity. We then examined their dentition, and only included participants with healthy dentition.

A surface electromyogram device (2EM-4D-MT; ReLive, 
Gimhae, Korea) was used to measure the masseter and temporalis muscles (Fig. 1). Two Ag/AgCl electrodes (Monitoring Electrode; 3M, Seoul, Korea) (diameter, $11.4 \mathrm{~mm}$; electrode frame diameter, $20 \mathrm{~mm}$ ) were placed on each muscle parallel to the direction of the muscle fibers, with the electrode centers separated by $2 \mathrm{~cm}$. ReLive 4D-MT software (version 3.3) was used to bandpass filter the signals between 25 and $500 \mathrm{~Hz}$, with a notch filter also applied to reduce powerline interference. Resting-state muscle activity, which was defined as reference voluntary contraction (RVC), was measured with the mandible in a resting position with the shoulders and waist straightened, lips lightly closed, and the tip of the tongue contacting the incisive papilla. Participants were instructed not to speak or swallow during this time. To measure the muscle activity during maximal biting, subjects were instructed to bite strongly in the centric occlusal position with the mouth closed. The examiner placed one hand on the subject's head and the other holding the subject's chin while applying a downward force to help measure muscle activity (Fig. 2). Normalized values of electromyogram signals were calculated by dividing the value of the maximal bite force by the value in the resting state for the same muscle, which we defined as percentage of RVC (\%RVC). Muscle activity was measured during the resting state and maximal biting three times for 5 seconds during each trial. The values for the middle 3 seconds were used.

In addition to surface electromyogram, we also observed the presence of the buccal mucosa ridge, tongue indentation, tooth cracks and fractures, and mandibular torus in the oral cavity. If a buccal mucosa ridge and tongue indentation were identified, the case was classified based on length in the molar region, extended to the premolar region, or throughout the buccal mucosa and the side of the tongue. Qraycam (Aiobio, Seoul, Korea) and Qraypen C (Aiobio) diagnostic equipment based on quantitative light-induced fluorescence were used to identify tooth cracks. The shape of the mandibular torus was first classified as bulging or lobulated, with the bulging shape being subdivided into a wide or narrow type according to the length of the base of the mandibular torus to the sum of the mesiodistal lengths of the mandibular first and second premolar. The lobulated shape was subdivided based on the number of lobules into single, double, and multiple types [25]. Next, a diagnostic model was produced to analyze the details of the mandibular torus structure, with the length and height measured using a digital caliper (Absolute Digimatic No. CD-20CPX; Mitutoyo, Kawasaki, Japan). All statistical analyses were conductedusing the software package SPSS (version 24.0; IBM Corp., Armonk, NY, USA). After testing for the normality of the activity values of the masseter and temporalis muscles, we further analyzed these values using Spearman's correlation coefficient and the nonparametric Mann-Whitney U test. Statistical significance was considered to be present at $P<0.05$.

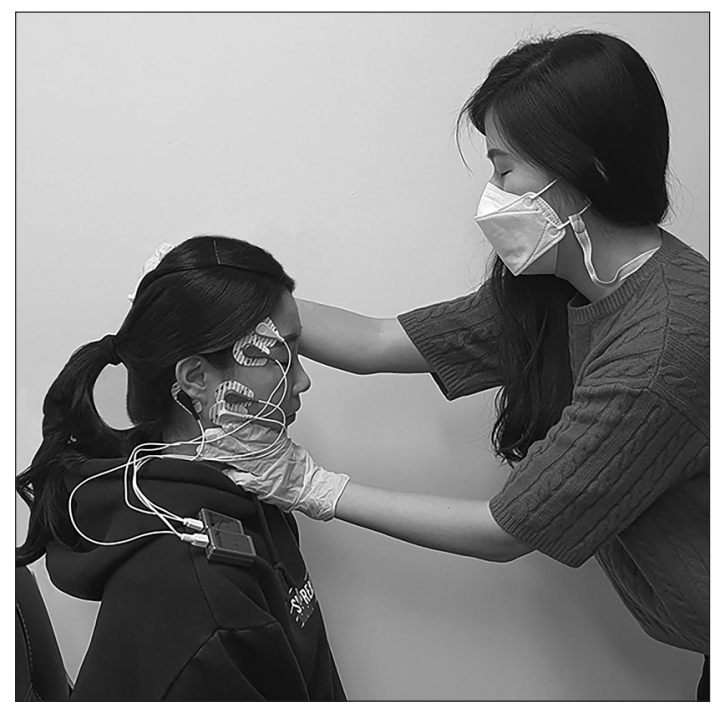

Fig. 2. Measurements of muscle activity during maximal biting.

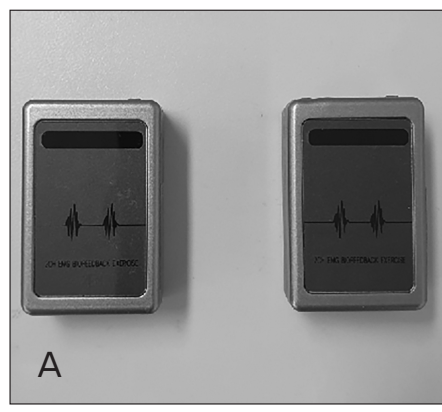

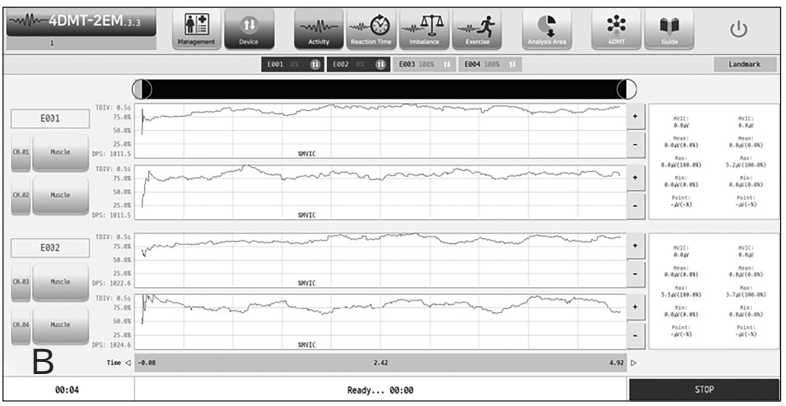

Fig. 1. (A) Surface electromyography equipment. (B) ReLive 4D-MT software (version 3.3). 
Table 1. General characteristics of the subjects

\begin{tabular}{|c|c|c|}
\hline Classification & Item & No. (\%) \\
\hline \multirow[t]{2}{*}{ TMD } & Presence & $10(33.3)$ \\
\hline & Absence & $20(66.7)$ \\
\hline \multicolumn{3}{|l|}{ TMD symptoms $(\mathrm{n}=10)$} \\
\hline \multirow[t]{2}{*}{ Pain in TMJ or muscles } & Presence & $3(30.0)$ \\
\hline & Absence & $7(70.0)$ \\
\hline \multirow[t]{2}{*}{ Articular sound during mandible movement } & Presence & $9(90.0)$ \\
\hline & Absence & $1(10.0)$ \\
\hline \multirow{2}{*}{ Limit of mandible movement } & Presence & $4(40.0)$ \\
\hline & Absence & $6(60.0)$ \\
\hline \multirow[t]{2}{*}{ Oral parafunctions } & Presence & $7(23.3)$ \\
\hline & Absence & $23(76.7)$ \\
\hline \multirow[t]{2}{*}{ Oral parafunctions sequela (headache or muscle fatigue) $(n=7)$} & Presence & $3(42.9)$ \\
\hline & Absence & $4(57.1)$ \\
\hline \multirow[t]{5}{*}{ Preference for hard and chewy food } & Never & $1(3.3)$ \\
\hline & Rarely & $5(16.7)$ \\
\hline & Neutral & $18(60.0)$ \\
\hline & Sometimes & $6(20.0)$ \\
\hline & Always & - \\
\hline \multirow[t]{3}{*}{ Direction when eating } & Left & $7(23.3)$ \\
\hline & Right & $14(46.7)$ \\
\hline & Both & $9(30.0)$ \\
\hline \multirow[t]{2}{*}{ Orthodontic treatment within the previous 2 years } & Yes & $10(33.3)$ \\
\hline & No & $20(66.7)$ \\
\hline
\end{tabular}

TMD, temporomandibular disorder; TMJ, temporomandibular joint.

Table 2. Buccal mucosa ridge and tongue indentation shape

\begin{tabular}{lc}
\multicolumn{1}{c}{ Classification } & No. $(\%)$ \\
\hline Length of the left buccal mucosa ridge $(\mathrm{n}=15)$ & $12(80.0)$ \\
Short & $1(6.7)$ \\
Medium & $2(13.3)$ \\
Long & \\
Length of the right buccal mucosa ridge $(\mathrm{n}=14)$ & $11(78.6)$ \\
Short & $1(7.1)$ \\
Medium & $2(14.3)$ \\
Long & \\
Length of the left tongue indentation $(\mathrm{n}=4)$ & - \\
Short & $1(25.0)$ \\
Medium & $3(75.0)$ \\
Long & \\
Length of the right tongue indentation $(\mathrm{n}=4)$ & - \\
Short & $1(25.0)$ \\
Medium & $3(75.0)$ \\
Long &
\end{tabular}

\section{Results}

\section{General characteristics}

Ten participants (33.3\%) experienced TMD and seven (23.3\%) experienced oral parafunction (bruxism or clenching), with mean durations of 1.2 and 0.9 years, respectively. Regarding eating habits, 18 (60.0\%) answered 'neutral' for preferring hard and chewy food, and 14 (46.7\%) chewed to
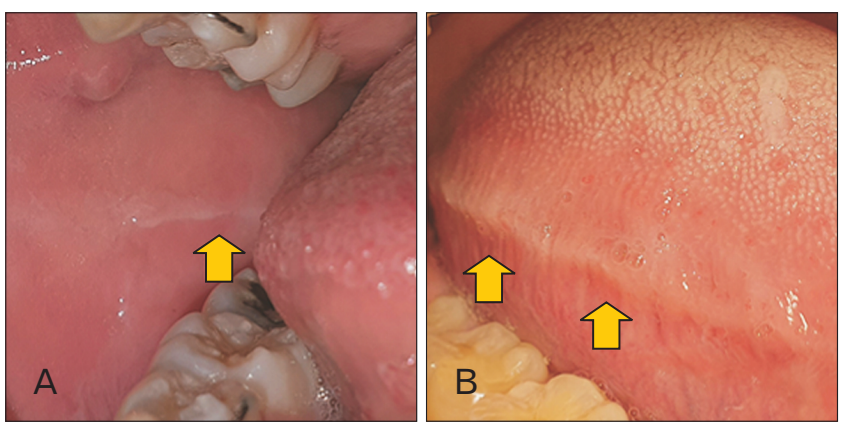

Fig. 3. Buccal mucosa ridge and tongue indentation (arrows). (A) Short-shape buccal mucosa ridge. (B) Long-shape tongue indentation.

the right while eating. Ten subjects (33.3\%) had received orthodontic treatment (Table 1).

\section{Activity of the masseter and temporalis muscles, and oral conditions}

The \%RVC values of the masticatory muscles were $10.39 \pm 4.31$ (mean \pm SD), $7.11 \pm 4.97,4.57 \pm 3.02$, and $2.83 \pm 1.34$ in the left masseter, left temporalis, right temporalis, and right masseter, respectively. A buccal mucosa ridge was observed in approximately half of all subjects, with 15 (50.0\%) and $14(46.7 \%)$ on the left and right, respectively, and on the 
left $(n=12,80.0 \%)$ and right $(n=11,78.6 \%)$ of the molar area only, which were the highest frequencies. Tongue indentation was observed in four subjects (13.3\%) on both sides, with three of them (75.0\%) having a long indentation along the side of the tongue (Table 2, Fig. 3). Tooth cracks and fractures were observed at 26 sites among 15 participants (50.0\%). Of the total sites, 15 sites (57.7\%) and 11 sites (42.3\%) were observed on the left and right sides, respectively. Furthermore, 17 of these sites $(65.3 \%)$ were observed in the mandibular first molar. Mandibular tori were observed in 17 (56.7\%) and $18(60.0 \%)$ subjects on the left and right sides, respectively. A narrow bulging shape of the mandibular torus was the most common, with eight (47.1\%) and nine (50.0\%) cases on the left and right sides, respectively (Table 3, Fig. 4).

\section{Correlations among duration of TMD, duration of oral parafunctions, size of the mandibular torus, and activity of the masseter and temporalis muscles}

The length of the left mandibular torus showed strong positive correlations with the length of the right mandibular torus and the heights of the left and right mandibular torus $(P<0.01)$. Significant positive correlations were also found between the length of the right mandibular torus, the heights of the left and right mandibular torus, the activity of the

Table 3. Mandibular torus shape

\begin{tabular}{|c|c|c|c|}
\hline Classification & Item & No. & Value \\
\hline \multirow[t]{2}{*}{ Mandibular torus length (mm) } & Left & 17 & $7.58 \pm 7.18$ \\
\hline & Right & 18 & $8.86 \pm 8.04$ \\
\hline \multirow[t]{2}{*}{ Mandibular torus height (mm) } & Left & 17 & $2.01 \pm 2.04$ \\
\hline & Right & 18 & $2.59 \pm 2.34$ \\
\hline \multirow[t]{5}{*}{ Left mandibular torus shape $(n=17)$} & Wide bulging & 4 & 23.5 \\
\hline & Narrow bulging & 8 & 47.1 \\
\hline & Single lobulation & - & - \\
\hline & Double lobulation & 5 & 29.4 \\
\hline & Multiple lobulations & - & - \\
\hline \multirow[t]{5}{*}{ Right mandibular torus shape $(n=18)$} & Wide bulging & 4 & 22.2 \\
\hline & Narrow bulging & 9 & 50.0 \\
\hline & Single lobulation & 2 & 11.1 \\
\hline & Double lobulation & 1 & 5.6 \\
\hline & Multiple lobulations & 2 & 11.1 \\
\hline
\end{tabular}

Values are presented as mean $\pm \mathrm{SD}$ or $\%$.
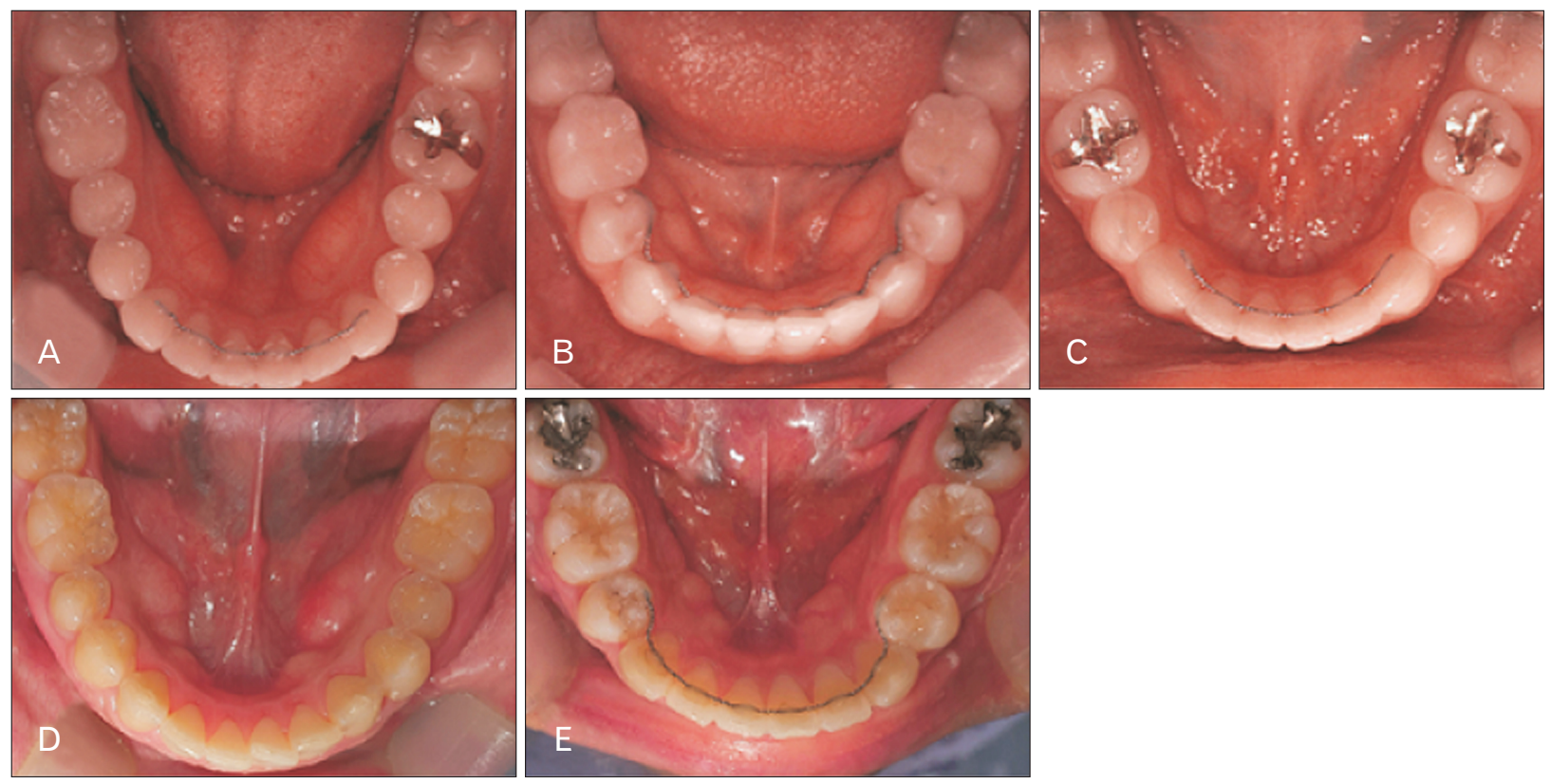

Fig. 4. Mandibular torus shape. (A) Wide bulging. (B) Narrow bulging. (C) Single lobulation. (D) Double lobulation. (E) Multiple lobulations. 


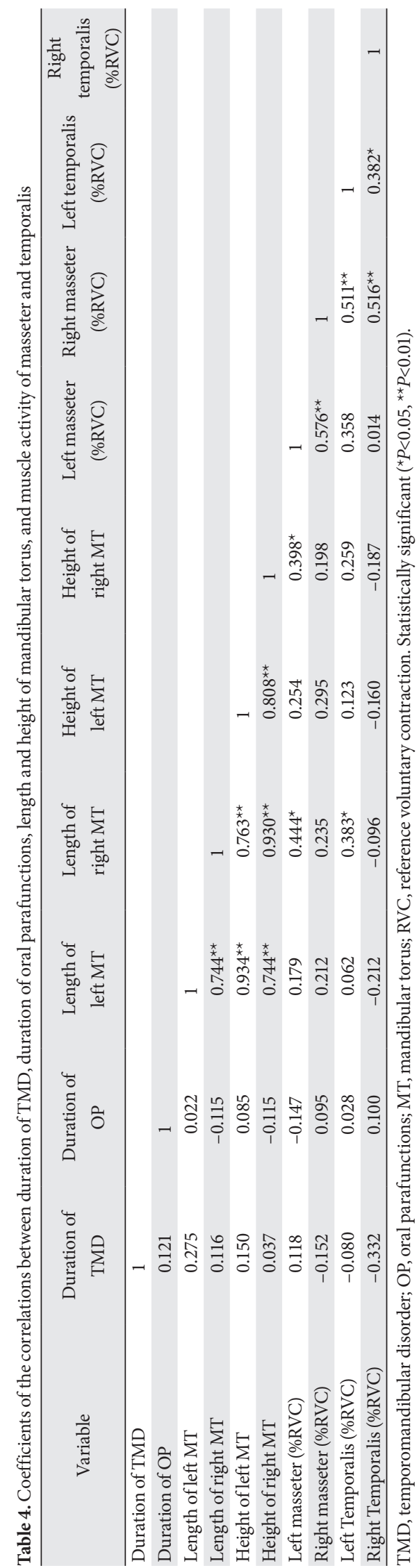

left masseter muscle, and the activity of the left temporalis muscle $(P<0.05)$. The height of the left mandibular torus had a strong positive correlation with that of the right mandibular torus $(P<0.01)$, and the height of the right mandibular torus had a significant correlation with the activity of the left masseter muscle $(P<0.05)$. Among the masticatory muscles, significant correlations were found between the activities of the left and right masseter muscles $(P<0.01)$, the right masseter muscle and both temporalis muscles $(P<0.01)$, and the left and right temporalis muscles $(P<0.05$; Table 4$)$.

\section{Comparison of the activity of the masseter and temporalis muscles according to the oral conditions}

The activity of the right temporalis muscle was higher in those without TMD than in those with this disorder $(P<0.05)$. There were differences in the activity of the left temporalis muscle in those with TMD who experienced restricted movement of the mandible $(P<0.05)$. The activity of the left masseter muscle was significantly higher in those who had not received orthodontic treatment $(P<0.05$; Table 5$)$. The group who did not have left buccal mucosa ridges exhibited more activity in the left masseter and less activity in the right temporalis muscle $(P<0.05)$. The activity of the left masseter muscle was significantly higher in those with no tongue indentation $(P<0.05$; Table 6$)$.

\section{Discussion}

The aim of this study was to obtain data for use in the dental field, which was achieved by analyzing masticatory muscle activity and oral conditions, and determining the relationships between these factors. Our study indicated that the muscle activity of the masticatory muscles was highest in the left masseter, followed by the left temporalis, right temporalis, and right masseter. The results of previous studies on the activity of the temporalis and masseter muscles are inconsistent [26]. The activity of the masticatory muscles may be inconsistent depending on the electromyogram electrode shape, age, or physical condition [27]. Bilateral observations were made for most of the buccal mucosa ridges, tongue indentations, and mandibular tori. Regardless of masticatory muscle activity, a short buccal mucosa ridge in the molar region, a long tongue indentation, and mandibular tori with a narrow bulging shape were the most common. Tooth cracks were often found in the mandibular first molar region. It can be predicted that this is because the mandibular first molar 
Table 5. Comparison of masseter and temporalis muscle activity according to TMD and related symptoms, oral parafunctions and related symptoms, and orthodontic treatment

\begin{tabular}{|c|c|c|c|c|c|c|c|c|c|c|c|c|}
\hline \multirow{3}{*}{ Item } & \multicolumn{6}{|c|}{ Masseter (\%RVC) } & \multicolumn{6}{|c|}{ Temporalis (\%RVC) } \\
\hline & \multicolumn{3}{|c|}{ Left } & \multicolumn{3}{|c|}{ Right } & \multicolumn{3}{|c|}{ Left } & \multicolumn{3}{|c|}{ Right } \\
\hline & Median & $Z$ & $P$ & Median & $Z$ & $P$ & Median & $Z$ & $P$ & Median & $Z$ & $P$ \\
\hline \multicolumn{13}{|l|}{ TMD } \\
\hline Presence $(n=10)$ & 16.10 & -0.264 & 0.792 & 12.50 & -1.320 & 0.187 & 13.70 & -0.792 & 0.428 & 11.00 & -1.980 & $0.048^{*}$ \\
\hline Absence $(n=20)$ & 15.20 & & & 17.00 & & & 16.40 & & & 17.75 & & \\
\hline \multicolumn{13}{|l|}{ TMD symptoms } \\
\hline \multicolumn{13}{|c|}{ Pain of TMJ or muscles } \\
\hline Presence $(n=3)$ & 14.67 & -0.173 & 0.863 & 7.00 & -1.763 & 0.078 & 8.00 & -1.555 & 0.120 & 11.00 & -0.933 & 0.351 \\
\hline Absence $(n=27)$ & 15.59 & & & 16.44 & & & 16.33 & & & 16.00 & & \\
\hline \multicolumn{13}{|c|}{ Articular sound during mandible movement } \\
\hline Presence $(n=9)$ & 17.11 & -0.656 & 0.512 & 13.00 & -1.018 & 0.309 & 13.33 & -0.882 & 0.378 & 11.11 & -1.788 & 0.074 \\
\hline Absence $(\mathrm{n}=21)$ & 14.81 & & & 16.57 & & & 16.43 & & & 17.38 & & \\
\hline \multicolumn{13}{|c|}{ Limit of mandible movement } \\
\hline Presence $(n=4)$ & 13.75 & -0.427 & 0.669 & 8.25 & -1.769 & 0.077 & 6.50 & -2.196 & $0.028^{*}$ & 11.75 & -0.915 & 0.360 \\
\hline Absence $(n=26)$ & 15.77 & & & 16.62 & & & 16.88 & & & 16.08 & & \\
\hline \multicolumn{13}{|l|}{ Oral parafunctions } \\
\hline Presence $(\mathrm{n}=7)$ & 12.86 & -0.907 & 0.364 & 16.71 & -0.417 & 0.677 & 15.14 & -0.123 & 0.902 & 17.43 & -0.662 & 0.508 \\
\hline Absence $(n=23)$ & 16.30 & & & 15.13 & & & 15.61 & & & 14.91 & & \\
\hline \multicolumn{13}{|c|}{ Oral parafunctions sequela } \\
\hline Presence $(n=3)$ & 13.67 & -0.380 & 0.704 & 9.33 & -1.279 & 0.201 & 10.33 & -1.072 & 0.284 & 9.00 & -1.348 & 0.178 \\
\hline Absence $(\mathrm{n}=27)$ & 15.70 & & & 16.19 & & & 16.07 & & & 16.22 & & \\
\hline \multicolumn{13}{|c|}{ Orthodontic treatment } \\
\hline Presence $(n=10)$ & 9.90 & -2.464 & $0.014^{*}$ & 14.70 & -0.352 & 0.725 & 16.40 & -0.396 & 0.692 & 16.20 & -0.308 & 0.758 \\
\hline Absence $(n=20)$ & 18.3 & & & 15.90 & & & 15.05 & & & 15.15 & & \\
\hline
\end{tabular}

TMD, temporomandibular disorder; TMJ, temporomandibular joint; RVC, reference voluntary contraction. Statistically significant $\left({ }^{*} P<0.05\right)$.

Table 6. Comparison of masseter and temporalis muscle activity according to the buccal mucosa ridge, tongue indentation, tooth cracks and fractures, and mandibular torus

\begin{tabular}{|c|c|c|c|c|c|c|c|c|c|c|c|c|}
\hline \multirow{3}{*}{ Item } & \multicolumn{6}{|c|}{ Masseter (\%RVC) } & \multicolumn{6}{|c|}{ Temporalis (\%RVC) } \\
\hline & \multicolumn{3}{|c|}{ Left } & \multicolumn{3}{|c|}{ Right } & \multicolumn{3}{|c|}{ Left } & \multicolumn{3}{|c|}{ Right } \\
\hline & Median & $Z$ & $P$ & Median & $Z$ & $P$ & Median & $Z$ & $P$ & Median & $Z$ & $P$ \\
\hline \multicolumn{13}{|l|}{ Buccal mucosa ridge } \\
\hline \multicolumn{13}{|l|}{ Left } \\
\hline Presence $(n=15)$ & 12.27 & -2.012 & $0.044^{*}$ & 15.20 & -0.187 & 0.852 & 15.27 & -0.145 & 0.885 & 19.00 & -2.178 & $0.029^{*}$ \\
\hline Absence $(n=15)$ & 18.73 & & & 15.80 & & & 15.73 & & & 12.00 & & \\
\hline \multicolumn{13}{|l|}{ Right } \\
\hline Presence $(n=14)$ & 13.00 & -1.455 & 0.146 & 15.21 & -0.166 & 0.868 & 14.36 & -0.665 & 0.506 & 18.36 & -1.663 & 0.096 \\
\hline Absence $(\mathrm{n}=16)$ & 17.69 & & & 15.75 & & & 16.50 & & & 13.00 & & \\
\hline \multicolumn{13}{|l|}{ Tongue indentation } \\
\hline \multicolumn{13}{|l|}{ Left } \\
\hline Presence $(n=4)$ & 6.50 & -2.196 & $0.028^{*}$ & 12.25 & -0.793 & 0.428 & 11.00 & -1.098 & 0.272 & 14.00 & -0.366 & 0.714 \\
\hline Absence $(n=26)$ & 16.88 & & & 16.00 & & & 16.19 & & & 15.73 & & \\
\hline \multicolumn{13}{|l|}{ Right } \\
\hline Presence $(n=4)$ & 6.50 & -2.196 & $0.028^{*}$ & 12.25 & -0.793 & 0.428 & 11.00 & -1.098 & 0.272 & 14.00 & -0.366 & 0.714 \\
\hline Absence $(n=26)$ & 16.88 & & & 16.00 & & & 16.19 & & & 15.73 & & \\
\hline \multicolumn{13}{|c|}{ Tooth cracks and fractures } \\
\hline Presence $(n=15)$ & 17.13 & -1.016 & 0.310 & 17.00 & -0.933 & 0.351 & 16.60 & -0.684 & 0.494 & 15.80 & -0.187 & 0.852 \\
\hline Absence $(n=15)$ & 13.87 & & & 14.00 & & & 14.40 & & & 15.20 & & \\
\hline \multicolumn{13}{|l|}{ Mandibular torus } \\
\hline \multicolumn{13}{|l|}{ Left } \\
\hline Presence $(n=17)$ & 16.41 & -0.649 & 0.517 & 17.24 & -1.235 & 0.217 & 16.53 & -0.732 & 0.464 & 14.35 & -0.816 & 0.414 \\
\hline Absence $(n=13)$ & 14.31 & & & 13.23 & & & 14.15 & & & 17.00 & & \\
\hline \multicolumn{13}{|l|}{ Right } \\
\hline Presence $(n=18)$ & 17.56 & -1.566 & 0.117 & 16.89 & -1.058 & 0.290 & 18.06 & -1.947 & 0.051 & 14.56 & -0.720 & 0.472 \\
\hline Absence $(n=12)$ & 12.42 & & & 13.42 & & & 11.67 & & & 16.92 & & \\
\hline
\end{tabular}

RVC, reference voluntary contraction. Statistically significant $\left({ }^{*} P<0.05\right)$. 
erupts at an early stage, and a problem such as dental caries increase the probability of restorative and prosthetic treatment. Furthermore, a relatively large force is applied to the corresponding tooth. However, to validate this conclusion it is still necessary to measure the occlusal force of each tooth area and perform additional investigations into the presence and type of restorations or prostheses.

In our study the average activity of the masticatory muscles was relatively low for those with TMD. In particular, when mandible movements were restricted, the average difference in the activity of the left temporalis muscle was larger. We observed that the activity of the left masseter muscle differed significantly according to whether or not orthodontic treatment had been performed within the previous 2 years. Our results also indicated a significant difference between the activities of the left masseter and the right temporalis muscles according to the presence or absence of a left buccal mucosa ridge: when this condition was present, the activity of the left masseter muscle was relatively low, and that of the right temporalis muscle was higher. We can infer that the activity of the agonist masticatory muscles decreased due to excessive activity over a long period.

The momentary force generated by a muscle increases when the muscle is properly rested [28]. Subjects who constantly chew the buccal mucosa or who eat over long periods develop buccal mucosa ridges, and they tend to exhibit lower activity in the masticatory muscles than those who do not have these ridges. In a previous study, after measuring the differences in pressure applied to the buccal mucosa surface of the posterior teeth area according to the presence or absence of the ridge, it was suggested that the pressure during biting and swallowing was significantly higher in the group with buccal mucosa ridges [29]. A study that analyzed the differences in pressure applied to the buccal mucosa and the lip mucosa in subjects with normal occlusion and malocclusion found that in the case of malocclusion, the pressure applied to the buccal mucosa also assists in inducing the formation of a buccal mucosa ridge [30]. It was also reported that appropriate positioning of the maxilla and mandible-rather than the number of teeth contacted during occlusion-is the most important factor for generating normal muscle activity during maximal biting [31]. To more accurately compare our results with previous studies, it is necessary to determine the presence and classification of malocclusion and also make pressure measurements of the buccal mucosa.

Previous studies on tongue indentation have indicated that the pressure exerted by the teeth on the surface of the tongue while resting, clenching, or swallowing does not induce tongue indentation. They also suggested that the resting width of the tongue plays an important role [30, 32]. In our study there were few cases of tongue indentation, but when it was present the activity of all masticatory muscles including the left masseter muscle was low. There was no difference in masticatory muscle activity based on the presence or absence of tooth cracks and fractures. We further found a correlation between the size of the right mandibular torus and the activity of the left masticatory muscles, whereas no significant difference was found in the activity of the masticatory muscles based on the presence or absence of a mandibular torus.

Our results cannot be generalized since the study was conducted on individuals in specific sex and age groups. There is a need to conduct an experiment with a large number of participants with more diverse characteristics. However, this study is valuable because it selected the number of subjects, sex, and age based on the results of relevant previous studies. One previous study analyzed the difference between masticatory muscle activity and bite force according to head posture and clenching level analyzed in 24 subjects [33]. Another study analyzed the relationship between craniofacial morphology and masticatory muscle activity in 40 participants [7], while the optimal signal bandwidth for recording the superficial electromyogram activities of the facial, jaw, oral, and neck muscles has been analyzed in 24 subjects [34]. Takagi and Sakurai [29] analyzed the factors related to the formation of the buccal mucosa ridge in 21 subjects. Therefore, the number of subjects in the present study was close to the average in previous studies.

Schmid-Schwap et al. [35] reported that females complained of TMD four times more often than did males, and that bruxism was more common in females. Many previous studies have obtained meaningful results from experiments only involving females, with most of these studies analyzing the associations between TMD, bruxism, and masticatory muscle activity under various conditions [36-40]. Other studies found that TMD symptoms and mandibular tori were much more common in young adults [41, 42]. Therefore, young female adults were selected as the subjects in the present study. If a comprehensive study is conducted by adding other factors related to oral conditions such as occlusal force and intraoral pressure, the relationship between masticatory muscle activity and oral conditions could be more clearly confirmed. The results of this study can provide 
basic data for improving the ability to prevent and diagnose diseases related to the masticatory muscles.

\section{ORCID}

Cha-Young Pyo: https://orcid.org/0000-0002-4447-2347

Tae-Hoon Kim: https://orcid.org/0000-0002-7930-9494

Da-Hye Kim: https://orcid.org/0000-0002-6684-7023

\section{Author Contributions}

Conceptualization: DHK. Data acquisition: CYP, DHK. Data analysis or interpretation: CYP, THK. Drafting of the manuscript: CYP, DHK. Critical revision of the manuscript: DHK, CYP. Approval of the final version of the manuscript: all authors.

\section{Conflicts of Interest}

No potential conflict of interest relevant to this article was reported.

\section{Acknowledgements}

This work was supported by the National Research Foundation of Korea (NRF) grant funded by the Korea government (MSIT) (No. 2020R1G1A1100960).

\section{References}

1. Carlsson GE, Droukas B. Dental occlusion and the health of the masticatory system. J Craniomandib Pract 1984;2:141-7.

2. Romero-Reyes M, Uyanik JM. Orofacial pain management: current perspectives. J Pain Res 2014;7:99-115.

3. Korean Oral Anatomy Research Group. Oral anatomy. Seoul: Komoonsa; 2017.

4. Kim TH, Kim DH. The effects of head rotation and tilt on oral pressure and muscle activity. Anat Cell Biol 2019;52:378-84.

5. Aidsman IK. Glossary of prosthodontic terms. J Prosthet Dent 1977;38:66-109.

6. Yoon HR, Choi YJ, Kim KH, Chung CR. Comparisons of occlusal force according to occlusal relationship, skeletal pattern, age and gender in Koreans. Korean J Orthod 2010;40:304-13.

7. Kim HB, Bae SS. Clinical application of electromyography and nerve conduction study. J Korean Phys Ther Sci 1998;5:603-16.

8. Takeuchi-Sato T, Arima T, Mew M, Svensson P. Relationships between craniofacial morphology and masticatory muscle activity during isometric contraction at different interocclusal distances. Arch Oral Biol 2019;98:52-60.
9. Custodio W, Gomes SG, Faot F, Garcia RC, Del Bel Cury AA. Occlusal force, electromyographic activity of masticatory muscles and mandibular flexure of subjects with different facial types. J Appl Oral Sci 2011;19:343-9.

10. Langlais RP, Miller CS, Gehrig JS. Color atlas of common oral diseases. 5th ed. Burlington: Jones \& Bartlett Learning; 2017.

11. Mizutani S, Ekuni D, Tomofuji T, Azuma T, Irie K, Machida T, Yoneda T, Iwasaki Y, Morita M. Factors related to the formation of buccal mucosa ridging in university students. Acta Odontol Scand 2014;72:58-63.

12. Piquero K, Ando T, Sakurai K. Buccal mucosa ridging and tongue indentation: incidence and associated factors. Bull Tokyo Dent Coll 1999;40:71-8.

13. Miyaura K, Matsuka Y, Morita M, Yamashita A, Watanabe T. Comparison of biting forces in different age and sex groups: a study of biting efficiency with mobile and non-mobile teeth. J Oral Rehabil 1999;26:223-7.

14. Al Quran FA, Al-Dwairi ZN. Torus palatinus and torus mandibularis in edentulous patients. J Contemp Dent Pract 2006;7:112-9.

15. De Luca Canto G, Torres de Freitas S, Schuldt Filho G, de Sousa Vieira R. Association between mandibular torus and parafunctional activity. J Stomat Occ Med 2013;6:43-9.

16. Pechenkina EA, Benfer RA Jr. The role of occlusal stress and gingival infection in the formation of exostoses on mandible and maxilla from Neolithic China. Homo 2002;53:112-30.

17. Reddy MS, Geurs NC, Wang IC, Liu PR, Hsu YT, Jeffcoat RL, Jeffcoat MK. Mandibular growth following implant restoration: does Wolff's law apply to residual ridge resorption? Int J Periodontics Restorative Dent 2002;22:315-21.

18. Shimomoto Y, Chung CJ, Iwasaki-Hayashi Y, Muramoto T, Soma K. Effects of occlusal stimuli on alveolar/jaw bone formation. J Dent Res 2007;86:47-51.

19. Fricton J. Temporomandibular disorders: a human systems approach. J Calif Dent Assoc 2014;42:523-33; discussion 531, 533 5.

20. Alkhalifah S, Alkandari H, Sharma PN, Moule AJ. Treatment of cracked teeth. J Endod 2017;43:1579-86.

21. Cameron CE. The cracked tooth syndrome: additional findings. J Am Dent Assoc 1976;93:971-5.

22. Okeson JP. Etiology of functional disturbances in the masticatory system. In: Okeson JP, editor. Management of Temporomandibular Disorders and Occlusion. St. Louis: Elsevier; 2013. p.130-63.

23. Speciali JG, Dach F. Temporomandibular dysfunction and headache disorder. Headache 2015;55(Suppl 1):72-83.

24. Rani S, Pawah S, Gola S, Bakshi M. Analysis of Helkimo index for temporomandibular disorder diagnosis in the dental students of Faridabad city: a cross-sectional study. J Indian Prosthodont Soc 2017;17:48-52.

25. Kim HS. The relationship between oral tori and temporomandibular joint symptoms and oral parafunctions [MS dissertation]. Seoul: Yonsei University; 2013.

26. Vitti M, Basmajian JV. Muscles of mastication in small chil- 
dren: an electromyographic analysis. Am J Orthod 1975;68:4129.

27. Ahlgren J, Sonesson B, Blitz M. An electromyographic analysis of the temporalis function of normal occlusion. Am J Orthod 1985;87:230-9.

28. Lee HJ, Lee YW. Correlation between cyclists' quadriceps femoris thickness and isokinetic strength. Korean J Sport 2019;17:1617-24.

29. Takagi I, Sakurai K. Investigation of the factors related to the formation of the buccal mucosa ridging. J Oral Rehabil 2003;30:565-72.

30. Gould MS, Picton DC. A study of pressures exerted by the lips and cheeks on the teeth of subjects with angle's class II division 1, clss II division 2 and class 3 malocclusions compared with those of subjects with normal occlusions. Arch Oral Biol 1968;13:527-41.

31. Tallgren A, Melsen B, Hansen MA. An electromyographic and roentgen cephalometric study of occlusal morphofunctional disharmony in children. Am J Orthod 1979;76:394-409.

32. Yanagisawa K, Takagi I, Sakurai K. Influence of tongue pressure and width on tongue indentation formation. J Oral Rehabil 2007;34:827-34.

33. Yang KY, Han KS, Yeo IS. Masticatory muscle activity and bite force according to head postureand clenching level. J Oral Med Pain 2003;28:249-59.

34. van Boxtel A, Boelhouwer AJ, Bos AR. Optimal EMG signal bandwidth and interelectrode distance for the recording of acoustic, electrocutaneous, and photic blink reflexes. Psychophysiology 1998;35:690-7.

35. Schmid-Schwap M, Bristela M, Kundi M, Piehslinger E. Sex- specific differences in patients with temporomandibular disorders. J Orofac Pain 2013;27:42-50.

36. Santana-Mora U, Cudeiro J, Mora-Bermúdez MJ, Rilo-Pousa B, Ferreira-Pinho JC, Otero-Cepeda JL, Santana-Penín U. Changes in EMG activity during clenching in chronic pain patients with unilateral temporomandibular disorders. J Electromyogr Kinesiol 2009;19:e543-9.

37. Raphael KG, Janal MN, Sirois DA, Svensson P. Effect of contingent electrical stimulation on masticatory muscle activity and pain in patients with a myofascial temporomandibular disorder and sleep bruxism. J Orofac Pain 2013;27:21-31.

38. Santiago V, Raphael K. Absence of joint pain identifies high levels of sleep masticatory muscle activity in myofascial temporomandibular disorder. J Oral Rehabil 2019;46:1161-9.

39. Wei F, Van Horn MH, Coombs MC, She X, Gonzales TS, Gonzalez YM, Scott JM, Iwasaki LR, Nickel JC, Yao H. A pilot study of nocturnal temporalis muscle activity in TMD diagnostic groups of women. J Oral Rehabil 2017;44:517-25.

40. Valentino R, Cioffi I, Vollaro S, Cimino R, Baiano R, Michelotti A. Jaw muscle activity patterns in women with chronic TMD myalgia during standardized clenching and chewing tasks. Cranio 2021;39:157-63.

41. Han DH, Park DY, Kim BI, Koh MY, Ahn YW, Kim JB. Global self-rating of oral health, concerns about oral health, and history of jaw injury related to temporomandibular joint symptoms in Korean adults. J Orofac Pain 2011;25:308-16.

42. Choi Y, Park H, Lee JS, Park JC, Kim CS, Choi SH, Cho KS, Chai JK, Jung UW. Prevalence and anatomic topography of mandibular tori: computed tomographic analysis. J Oral Maxillofac Surg 2012;70:1286-91. 